\title{
Les apories du surréalisme
}

\author{
Boštjan Marko Turk
}

\section{Synopsis}

La présente étude se propose de répondre à la question des apories qui concernent la conception surréaliste du langage et de ses outils poétiques. Les surréalistes démettaient du premier rang la disposition d'esprit d'après laquelle ce qui existe possède sa raison d'être et peut - par conséquent - être considéré comme intelligible. Ils postulaient qu'il y avait une sphère au-delà de la réalité dans laquelle la raison nétait pas capable de pénétrer. Or, tous les outils que les surréalistes proposaient afin d'atteindre la surréalité étaient conçus sur les modalités du raisonnement. Ainsi, ils se sont laissés piéger par leur propre piège.

Mots-clefs : surréalisme, raison, pensée, esprit, Descartes, Voltaire, Breton, langue, linguistique, signe, Benveniste, Saussure, antinomie. 
La définition du surréalisme qu'a proposée André Breton permet de bien déceler ses options fondamentales :

«Automatisme psychique pur par lequel on se propose d'exprimer, soit verbalement, soit par écrit, soit de tout autre manière, le fonctionnement réel de la pensée. Dictée de la pensée, en l'absence de tout contrôle exercé par la raison, en dehors de toute préoccupation esthétique ou morale » (Breton 1924, 12).

Grâce à cette formule, il est possible d'indiquer avec précision deux éléments qui cernent les traits pertinents du mouvement. Au premier plan, il est question de déni intégral de la rationalité et des principes idéaux servant de référence aux membres de la communauté universelle qui se fondent conformément - sans exception ni réserves - sur cette faculté. Il s'agit alors de contester le statut de la pensée en tant quélément constitutif de l'identité de l'Occident. Le surréalisme n'est pas uniquement l'antithèse de la pensée et de son emprise sur la réalité rendue possible par l'appareil relatif à la connaissance et permettant au sujet de l'acquérir, tel qu'il s'est formé dans l'histoire de la pensée. Il est l'antithèse de tout ce que la pensée transmet, implique et connote; il est la désapprobation de la pensée et ce qui plus est : le rejet de tout mode de penser qui procéderait à la prise de la réalité de la façon réfléchie : spontanée mais aucunement automatique et en dehors du contrôle de la logique. Comme on n'a pas vu une chose pareille dans l'histoire de l'Occident, cette prise de position s'avère lourde de conséquences.

Le postulat «le fonctionnement réel de la pensée» présuppose donc qu'une formation de la pensée, soumise à ce qui a été communément accepté comme la dialectique cartésienne procédant des quatre règles méthodologiques, ${ }^{1}$ ne soit pas suffisante. De plus, la postulation inaugurale de l'ouvrage où la nouvelle approche a été proposée : «Le bon sens est la chose du monde la mieux distribuée» (Descartes 1966, 33) se voit, elle aussi, dépourvue de l'envergure nonobstant son caractère général qui établissait la formule au niveau des vérités irrécusables ne pouvant ainsi pas être contredites.

Néanmoins, la vraie «pensée» surréaliste ne devrait être créée qu'en totale indépendance de l'objet de la pensée et des modalités qui la conditionnent. Les surréalistes prenaient de grands airs la disposition d'esprit d'après laquelle ce qui existe possède sa raison d'être et peut - par conséquent - être considéré comme intelligible. Le surréalisme eut peur de l'intelligible. Ses adhérents invoquaient que la raison ne saurait être la source de la connaissance et contestaient sa légitimité concernant les moyens de l'appréhension dont le soubassement est la faculté propre à l'être humain de penser, de connaître et de juger. C'est celle qui se présente sous le terme de «la raison» : nominalement c'est ce qui permet de distinguer le genre homo sapiens sapiens des autres, dans l'univers du vivant :

1 Id est : le doute, la division, la synthèse et la vérification. 
«Pour nous, jeunes surréalistes de 1924, la grande «prostituée», c'était la raison. Nous jugions que cartésiens, voltairiens et autres fonctionnaires de l'intelligence, ne l'avait fait servir qu'à la conservation des valeurs à la fois établies et mortes, tout en affectant un non-conformisme de façade. Et, en accusation suprême, de lui avoir donné pour besogne mercenaire de persifler «l'Amour, la Poésie». Cette dénonciation fut faite par notre groupe avec une extrême vigueur» (Masson 1976, 16-17).

André Breton allait encore plus loin lorsqu'il avançait que la logique devrait être démise du rang qu'elle occupait et reléguée parmi les outils secondaires. La logique, sous-entendue par le chef du groupe surréaliste, doit être étymologiquement entendue dans le sens qu'il recouvre l'ensemble de ses acceptations. Le terme «la logique» vient du mot «logos». Celui-ci indique le discours : comme le discours via pars pro toto reflète la capacité de produire des signes linguistiques, ceux-ci s'exprimant au niveau des messages organisés, au niveau de la syntaxe, le logos connote la langue. Comme celle-ci ne peut être organisée que d'une façon logique, la langue équivaut à la rationalité. Dans la philosophie grecque - dont le christianisme, l'assise de l'esprit occidental, hérita - le logos est considéré comme la raison de tout ce qui existe contenant en soi des germes ontologiques de l'existence d'autre chose.

Par là, le mot «logique» rejoint la formulation cartésienne du «bon sens», au moins en ce qui concerne le caractère général de cette notion : la rationalité est l'anabasis, la clef universelle qui permet de se procurer l'essence des choses qui existent, d'appréhender leur nature intelligible et de soumettre ses éléments aux actes intellectuels du jugement analytique. De ce point de vue, le grief contre le «bon sens» (Descartes 1966, 33) qui termine « l'appel » d'André Breton ne devrait pas surprendre :

«Nous vivons encore sous le règne de la logique, voilà, bien entendu, à quoi je voulais en venir. Mais les procédés logiques, de nos jours, ne s'appliquent plus qu'à la résolution de problèmes d'intérêt secondaire. Le rationalisme absolu qui reste de mode ne permet de considérer que des faits relevant étroitement de notre expérience. ${ }^{2}$ Les fins logiques, par contre, nous échappent. Inutile d'ajouter que l'expérience même s'est vu assigner les limites. Elle tourne dans une cage d'où il est de plus en plus difficile de la faire sortir. Elle s'appuie, elle aussi, sur l'utilité immédiate, et elle est gardée par le bon sens» (Breton 1924, 18-19).

Voulant davantage dégrader le domaine de l'intelligible, Breton passe à argumenter l'insuffisance de la raison qui ne saurait fournir que «des faits relevant étroitement de notre expérience» ce qui le ramène dans la contradiction : en se servant d'une nouvelle analogie pour montrer les faiblesses du raisonnement, il confronte

2 Cité supra. 
celui-ci au terme de l'image, celle-ci lui étant, d'après André Breton, nettement supérieure. Or, pour le démontrer, il remet - à son insu - la même image au rang de l'empirisme, c'est-à-dire, au niveau des faits qui servent d'expérience. Le seul enseigne des compétences intellectuelles (le synonyme de la notion «esprit» dont il se sert) serait la faculté qui est reliée «aux problèmes d'intérêt secondaire». Les étincelles et le réseau des idées qu'elles évoquent n'appartiennent qu'au niveau de l'expérience puisqu'elles ne sont perceptibles que par les sens :

»Et de même que la longueur de létincelle gagne à ce que celle-ci se produise à travers des gaz raréfiés, l'atmosphère surréaliste créée par l'écriture mécanique, que j'ai tenu à mettre à la portée de tous, se prête particulièrement à la production des plus belles images «. (Breton 1924, 12).

Qu'est-ce-que l'étincelle, substitut extralinguistique de l'image sinon un «des faits relevant étroitement de notre expérience» ?3 L'auteur devrait en être conscient, d'autant plus que l'image surréaliste n'est définie que par les termes qui concernent l'expérience. Il appartient toujours au niveau du vécu, c'est donc un fait perçu nominativement. Fait parallèle à cette mise en évidence personnelle, le texte suivant qui met en relief l'expérience comme la clef de toute appréhension de l'outil fondamental du mouvement : «Avec la modernité, que les futurs surréalistes ont déjà découverte chez Apollinaire, la sincérité, qui donne à sa poésie la force poignante de l'expérience vécue "(Légoutière 1972, 36).

L'antinomie s'approfondit dans le texte suivant qui est anthologique en ce qui concerne le rapport entre l'image et l'esprit. ${ }^{4}$ L'image guiderait l'esprit ; celui-ci s'alimentant par les sens, par l'expérience. Hausser les images au-dessus de la raison, c'est suspendre (contredire) leur existence parce que c'est l'expérience qui les fournit à l'entendement. A ne pas parler du fait que les images, dans leur complexité, ne pourraient exister que par la raison et en raison, «bon sens» comme l'appelle Descartes. C'est la garantie de leur «compréhensibilité» par n'importe quel auditoire ou lectorat. Le réseau identitaire entre «l'image», «l'expérience» et «l'esprit» est soumis au contresens, à un arbitraire libéré de toute entrave en ce qui concerne les normes du discours :

» On peut même dire que les images apparaissent, dans cette course vertigineuse, comme les seuls guidons de l'esprit. Lesprit se convainc peu à peu de la réalité suprême de ces images. Se bornant d'abord à les subir, il s'aperçoit bientôt qu'elles flattent sa raison, augmentent d'autant sa connaissance. Il prend conscience des étendues illimitées où se manifestent ses désirs, où le pour et le

\footnotetext{
3 Cité supra.

4 L'entendement égale l'esprit, à voir la définition du mot in fine.
} 
contre se réduisent sans cesse, où son obscurité ne le trahit pas. Il va, porté par ces images qui le ravissent, qui lui laissent à peine le temps de souffler sur le feu de ses doigts « (Breton 1924, 12).

Or, ce serait se méprendre sur l'essentiel que de penser que les postulats de l'illogisme que les surréalistes mettaient en relief ciblaient les analyses formelles de la connaissance et le raisonnement que proposaient la philosophie des lumières et celle du rationalisme, notamment la pensée de Voltaire et celle de Descartes. L'ambition du groupe visait plus loin que cela, quoi qu'implicitement. Se voulant instrument de connaissance, le surréalisme se confrontait, à son insu, aux antinomies que le nouveau mode de «penser» nétait pas capable d'envisager, ni de s'en rendre compte, à vrai dire. Ce qui manquait au surréalisme c'étaient les exigences de l'esprit spéculatif. Faute de ça les surréalistes voulant écarter les conventions de la vérité ontologique du langage, telles que les différentes écoles les ont introduites dans la pensée et la civilisation occidentale, se sont heurtés inévitablement aux postulats fondamentaux sans lesquels il n'y pas de pensée, ni occidentale ni une autre.

Ils sont passés outre aux axiomes qui permettent à toute pensée de naître et de s'exprimer comme telle. L'avis critique mettait en question plus que la philosophie dualiste et rationaliste représentée - dans les yeux d'un Français : et les surréalistes étaient français et ne raisonnaient qu'à la française - par Descartes et de Voltaire. La critique ambitionnait davantage et ce qu'elle envisageait comme "problèmes d'intérêt secondaire» était, en fait, un problème du premier plan, une réalité, a parte ante.

Les surréalistes ne pouvant pas entendre la pensée en tant que telle eurent recours à la série d'accidents circonstanciels par lesquels ils tâchaient de redéfinir «la pensée» en la ployant à toutes sortes de circonstances et de modifications bien que cela ait entraîné des paralogismes. La pensée est un phénomène univoque : son statut ontologique empêche de lui donner des épithètes : il n'y a pas d'épithète non plus concernant le verbe être. Lorsque les surréalistes décidèrent de traiter «la pensée» dans le sens équivoque, la pensée qu'ils envisageaient nétait plus la pensée dans le sens propre du terme. Il n'y a pas de pensée qui fonctionnerait «réellement» comme il n'y a pas de pensée qui serait «dictée» en l'absence de tout contrôle exercé par la raison, en dehors de toute préoccupation esthétique ou morale» (Breton 1924, 12). $\mathrm{Si}$ on astreint la pensée à un mode pareil des aléas, elle cesse d'exister. Les circonstances que les surréalistes stipulaient afin que la "pensée» existe, n'existaient pas, au moins dans le sens de permettre à la pensée d'exister. C'est une antinomie. Il n'y a pas des pensées, il y a la pensée.

Le raisonnement surréaliste s'avère donc un apriorisme mal fondé surtout si on considère que les surréalistes se définissaient avant tout comme des intellectuels : quelle serait donc cette intellectualité privée de la pensée intelligible : "Donc envisageant l'origine est la portée du surréalisme comme le mouvement intellectuel spontané, 
nous sommes amenés à reconnaître qu'il ne peut désormais s'engager que dans deux directions» (Naville, 1975, 76). L'aporie tend à s'approfondir davantage étant donné le fait que la famille d'esprit surréaliste est communément perçue dans le sens de former une école philosophique présentant un courant autonome de pensée : «Au sens le plus étroit, le surréalisme est un procédé d'écriture, au sens large une attitude philosophique qui est en même temps une mystique (ou qui le fut), une poétique, et une politique» (Raymond 1940, 282). De plus : comment faire «de la politique» «en l'absence de tout contrôle exercé par la raison, en dehors de toute préoccupation esthétique ou morale " (Breton 1924,12) est une question per se. Quelle serait la politique qui serait conçue «en dehors de toute préoccupation morale»? Le siècle d'André Breton en a, pourtant, fourni quelques modèles néfastes.

Il n'y notamment pas de pensée en dehors de la langue et il n'y pas de langue en dehors des catégories linguistiques, basées sur la prédication et présupposant lobjet de toute pensée. Il n'y a pas de pensée sans objet ; la pensée reliée par la prédication à son complément requiert l'objet : elle ne peut pas exister autrement. Le lien reliant les membres du syntagme originel est celui qui a permis - à un moment donné - l'évolution de l'homme moderne. Le cartésianisme et le voltairianisme sont des phénomènes ex post; on pourrait volontiers les définir comme d'intérêt secondaire» par rapport aux propositions posées a priori. Ainsi :

«C'est pourquoi Heidegger insiste tout autant que Benveniste sur cette fusion entre fonction et sens qu'il comprend évidemment, non comme obstacle accidentel à l'intelligibilité linguistique, mais comme abri historial de la multiplicité, rappel de «la façon dont les Grecs comprenaient l'essence de lêtre» (Heidegger 1967, 100)» (Cassin 1998, 28).

L'homme est un être de l'intelligible : la logique qui organise le langage (dans l'aperception saussurienne du mot) est le pivot autour duquel tournent les événements ontologiques qui lui sont propres. Ce n'est rien ce que Voltaire et Descartes auraient conçu : c'est une prédisposition qui est propre à l'homme, depuis ses origines :

«C'est alors, dans un temps indécidable de l'histoire ou de l'origine qu'interfèrent le syntaxique et le sémantique, le grammatical et le lexical, et que déteignent l'un sur l'autre le «est» de la logique et le est de réel. La fonction assertive, précipitable en sens véritatif, le montrait déjà par cette hybridation même »(Cassin 1998, 26).

Dénoncer «la grande 'prostituée»", la raison» et mettre au banc des accusés les fonctionnaires de l'intelligence " ${ }^{5}$ ainsi que «la logique» ${ }^{6}$ est lourd de conséquences,

5 Cité supra.

6 Cité supra. 
puisque l'histoire de la pensée se présente en tant que domaine de l'intelligible, tout processus mental dont l'équivalence étaient les opérations de l'intelligence ou l'usage du «bon sens, la chose la mieux distribuée du monde» (Descartes 1966, 33) fut existant, avant l'invention de la représentation, à l'aide de signes graphiques établis de façon conventionnelle, de la parole, de la pensée. La parole et la pensée naissent avec l'homme : comme les surréalistes étaient de «genus» de l'espèce qui ne différait de celui dont la définition se réduit au genre animal, capable de se servir du code sémiotique et de transmettre ainsi la culture, ils ne pouvaient pas ignorer les étapes évolutives dans le devenir de l'homme dont ils étaient la dernière flambée, si on adopte le point de vue diachronique en se mettant à l'actualité de l'année 1924. La «révolution surréaliste» visait le surréalisme lui-même et menaçait de supprimer, d'une façon plus qu'efficace, l'arsenal de ses armes puissantes, parmi lesquelles l'expérience de communiquer en signes linguistiques et de se servir du triplet formé du signifié, du signifiant et de ses règles d'emploi. Le lien entre les trois notions, ainsi que la logique syntaxique étaient toujours là : il a permis la naissance de l'articulation intellectuelle, dans la multiplicité de ses apparences, depuis l'avènement de l'homme ou de l'invention de l'écriture. Le mouvement surréaliste fait partie de l'ensemble quoiqu'il y refuse l'allégeance.

Lorsque le «temps indécidable de l'bistoire ou de l'origine» (Cassin 1998, 28) se concrétise et fait apparaître les premiers penseurs qui passent à l'élaboration des systèmes, les règles qui régissent les représentations, ${ }^{7}$ soit abstraites soit concrètes, «des phénomènes de l'univers» étaient disponibles de même façon qu'ils le sont aujourd'hui. La civilisation de la parole est redevable aux Grecs, premièrement :

«Le fait que la formation de la grammaire occidentale soit due à la réflexion grecque sur la langue grecque donne à ce processus toute sa signification. Car cette langue est, avec l'allemand, au point de vue des possibilités du penser, à la fois la plus puissante de toutes et celle et le plus la langue de l'esprit » (Heidegger 1967, 67).

C'est une phrase à l'intérieur de laquelle on substituerait facilement une langue à une autre (l'allemand pourrait être, $e g$. remplacé par le français, la langue plus complète), le message de l'existentialiste ne subirait, pourtant, aucun changement.

Aristote est celui qui a procédé à l'analyse de l'ontologie de la langue, le moyen d'action et l'instrument sine qua non de tout transfert linguistique. ${ }^{8}$ Ainsi :

$7 \quad$ La représentation implique la communication.

8 Le texte suivant est extrait du sixième chapitre du livre Problèmes de linguistique générale, intitulé : «Catégories de pensée est catégories de la langue». Sa référence principale est la pensée d'Aristote concernant la langue et ses catégories. 
«Cette grande structure, qui enferme des structures plus petites et de plusieurs niveaux, donne sa forme au contenu de pensée. (...) Ce contenu doit passer par la langue et en emprunter les cadres. Autrement, la pensée se réduit sinon exactement à rien, en tout cas à quelque chose de si vague et de si indifférencié que nous n'avons aucun moyen de l'appréhender comme «contenu» distinct de la forme que la langue lui confere. La forme linguistique est donc non seulement la condition de transmissibilité, mais d'abord la condition de la réalisation de la pensée. Nous ne saisissons la pensée que déjà appropriée aux cadres de la langue» (Benveniste 1968, 64).

Benveniste reprend la logique cartésienne : il établit le lien identitaire entre la forme linguistique et la pensée. Par la suite il postule résolument :

«C'est à dire que la question de savoir si la pensée peut se passer de la langue ou la tourner comme un obstacle, pour peu qu'on analyse avec rigueur les données en présence apparaît dénuée de sens» (Benveniste, 1968, 64).

Le couplet « la forme linguistique» et «la pensée» est corroboré par l'introduction de tertium comparationis sans lequel ne sont possibles ni la pensée ni son équivalent linguistique, c'est l'objet de la pensée. Ce sont les classes dans lesquelles on répartit chacun des genres les plus généraux de l'actualité afin de pouvoir organiser l'expérience. A travers la substance qui se propose les modes d'exister dont la liste est close ${ }^{9}$ la pensée s'ancre dans la réalité telle que la raison la perçoit. Notamment, la citation suivante a le caractère d'une amère leçon quant au surréalisme :

«Or, nous avons la bonne fortune de disposer de donnés qu'on dirait prêtes pour notre examen, élaborées et présentées de manière objective, intégrées dans un ensemble connu : ce sont les catégories d'Aristote. Il nous sera permis de considérer ces catégories comme la liste des concepts a priori qui, selon lui, organisent l'expérience. C'est un document de grande valeur pour notre propos» (Benveniste 1968, 65).

À charge de revanche, Benveniste, sur le chemin de l'ontologie, relit les catégories du Philosophe à la forme comme réalité intelligible immanente à la matière sensible et à la pensée, relevant de l'expérience. Rien dans l'intelligible n'existe en dehors de la logique. Les catégories reprises par Benveniste d'après Aristote, ont pour soubassement la logique, puisque l'être même est logique : il ne saurait exister sans celle-ci et vice versa

Les apories de l'approche surréaliste revêtent le caractère insurmontable lorsque la faculté qui remplacerait la raison est en jeu. Le surréalisme postu-

9 Ce sont : substance, quantité, qualité, relation, lieu, temps, position, avoir, agir, subir, bref toutes les modalités et propriétés de l'être que la raison humaine peut concevoir. 
lait l'imagination qui se substituerait à l'intelligible. Pourtant l'imagination n'est autre chose qu'un des dérivés de la pensée consciente et rationnellement organisée. L'imagination est le pouvoir de se représenter ou de former des images à travers l'esprit, à partir des éléments qui proviennent de perceptions sensorielles ou bien sont formés via abstrahendi. Evolutivement, elle est l'outil de la conscience réflexive née du besoin de résoudre les problèmes par l'utilisation de la simulation mentale.

Il existe deux sortes d'imagination, active et passive. La première apparaît sous la forme de l'activité cognitive : son but est de réassembler différents souvenirs (ou impressions) pour former une nouvelle représentation dont se servira l'esprit. La deuxième apparaît quand l'esprit se représente involontairement les impressions sensibles. L'imagination et les images comme son produit, bien qu'enveloppées par les fervents du mouvement dans un langage abscons - au moins pour les profanes - se reconnaissent unanimement dans les termes, repris d'après Le Dictionnaire de l'Académie française $e^{10}$ et exposés supra. Ce sont les surréalistes qui l'admettent en premier.

"La beauté de l'image, sa "lumière» tient à la fulgurance d'une révélation. L'esprit d'abord ébloui se convainc peu à peu de la réalité suprême de ces images (Breton 1924, 52)» (Abastado 1971, 83). Et, dans l'espace du même paragraphe : «Cette connaissance peut être conçue comme subjective ou objective, témoignage sur la psyché ou sur le monde, selon que l'esprit est passif ou actif» (Abastado 1971, 83). Encore plus relevant est le texte qui concerne le programme du mouvement, celui de Louis Aragon :

«Cette peur de l'erreur, que dans la fuite de mes idées tout, à tout instant me rappelle, cette manie de contrôle, fait préférer à l'homme l'imagination de la raison à l'imagination des sens. Et pourtant c'est toujours l'imagination seule qui agit. Rien ne peut m'assurer de la réalité, rien ne peut m’assurer que je la fonde sur un délire d'interprétation, ni la rigueur d'une logique ni la force d'une sensation. Mais dans ce dernier cas l'homme qui en a passé par diverses écoles séculaires s'est pris à douter de soi-même : par quel jeu de miroirs fût-ce au profit de l'autre processus de pensée on l'imagine» (Aragon 1928, 12).

Ce qui est surprenant, c'est que Louis Aragon mette en œuvre le mécanisme cartésien du doute qu'il paraît pasticher du texte où le philosophe en parle. Dans les Méditations métaphysiques, Descartes, afin d'établir le socle de la gnoséologie, chasse notamment les «délires d'interprétation» (Aragon 1928, 12) que pourrait imposer «un certain mauvais génie» (Descartes 2011, 67). Par là le philosophe fonde l'école du doute : la lignée rationaliste qui institue le cogito et par cela établit l'autonomie du sujet. Le cogito cartésien est libéré par le doute qui replace le sujet

10 Cf : https://www.dictionnaire-academie.fr/article/A9I0182, consulté le 27 juillet, 2019. 
dans l'individualisme de la modernité où il ne dépend que des décisions qui émanent de «soi».

Or, la liberté et le statut de l'indépendance sont ce que revendiquaient les surréalistes lorsqu'ils requéraient un sujet affranchi de toute entrave, surtout de celle des contraintes de la pensée, oblitérée et faussée par le prétendu despotisme du raisonnement occidental. En fait : le surréalisme a profité du sujet ${ }^{11}$ qui a été davantage libéré par la révolution gnoséologique de l'époque rationaliste. Celle-ci se résumait au doute cartésien, indissociable des opérations mentales qui ont - en conséquence - institué le suppositus de toute opération se réclamant de caractère «surréaliste». Si on paraphrasait Aragon on mettrait de la sorte : "Voltaire et Descartes sont surréalistes au même point que Stagrite et Benveniste»... Parce que, si on cherchait un mot qui reprendrait efficacement l'entreprise, on hésiterait entre «la négation» et, surtout, «le doute».

En conséquence, le sujet cartésien et celui des surréalistes se rencontrent dans une étrange coïncidence. Celle-ci est logique parce que les objectifs de la philosophie de cogito et ceux de l'individuation de la conscience autonome, susceptible des procédés jusqu'à alors inouïs ${ }^{12}$, sont concomitants. Il ne faut que considérer les uns et les autres dans l'optique à laquelle est conforme le présent écrit. Ceci n'a pourtant rien de surprenant : depuis l'invention de l'écriture, signe métonymique de l'identité de l'homme moderne, les énigmes analogues tourmentaient ceux qui voulait apporter une réponse aux arcanes de la langue : les approches et les résultats des recherches ne pouvaient pas différer essentiellement les uns des autres, puisque la matière sur laquelle s'imposaient les exercices fut uniforme et commandait le chemin de recherche dans les sens apparentés. Une lecture comparée des textes relève notamment l'enjeu de la coïncidence évoquée :

«Je supposerai donc qu'il y a, non pas que Dieu, qui est très bon et qui est la souveraine source de vérité, mais qu'un certain mauvais génie, non moins rusé et trompeur que puissant, a employé toute son industrie à me tromper. Je penserai que le ciel, l'air, la terre, les couleurs, les figures, les sons et toutes les choses extérieures que nous voyons, ne sont que des illusions et tromperies, dont il se sert pour surprendre ma crédulité. Je me considérerai moi-même comme n'ayant point de mains, point d'yeux, point de chair, point de sang, comme n'ayant aucun sens, mais croyant faussement avoir toutes ces choses. Je demeurerai obstinément attaché à cette pensée ; et si, par ce moyen, il n'est pas en mon pouvoir de parvenir à la connaissance d'aucune vérité, à tout le moins il est en ma puissance de suspendre mon jugement» (Descartes 2011, 67).

11 Le sujet dans le sens de ce (celui) qui est le siège des événements ontologiques, c'est-à-dire, la personne humaine.

12 Id est : écriture automatique et la mécanique des images. 
À confondre l'entendement, l'image surréaliste, produit de l'imagination, provenant « des profondeurs de notre esprit» (Breton 1924,5) - et obtenue par le processus de l'écriture automatique - se compose des éléments que l'esprit ne peut saisir qu'à l'intérieur du paradigme se fondant sur l'intelligible. La structure de l'image est en conformité avec la dénomination de l'entité du premier niveau quant à l'articulation sémiologique : l'image est - du point de vue de l'ontologie du signe - une entité bipartite. Elle dénote une «chose» en dehors du langage par les moyens du langage articulé. L'image surréaliste confirme notamment l'appréhension structuraliste sur les rapports étroits entre la science de la langue et celle des signes. Aux yeux de Ferdinand de Saussure la linguistique nétait qu'une branche de la sémiologie, la branche toutefois la plus développée, en raison de la complexité du langage. Que l'image soit un signe ultra complexe, les surréalistes le savaient parmi les premiers puisqu'ils tenaient à faire d'elle un outil pour décrypter les messages codés : une langue se servira des signes linguistiques qu’on appelle communément les mots : à l'aide de ceux-ci on découvre - en les nommant ${ }^{13}$ - les entité de l'univers extralinguistique. «Breton veut voir en image un moyen de déchiffrer le monde qui s'offre à l'esprit comme cryptogramme» (Abastado 1971, 83).

Aux yeux d'André Breton, l'image serait un outil de l'intelligence : «déchiffrer» équivaut notamment à comprendre ce qui se présente de façon peu distincte ou difficilement intelligible. Le fondateur du mouvement parait intrigué par l'intelligible : Ferdinand Alquié s'est, à propos de l'ambigüité intrinsèque exprimé de la sorte ${ }^{14}$ :

«Breton reprend à son compte une théorie qui domina l'enseignement philosophique jusqu'aux environs de 1935, théorie selon laquelle l'imagination est une faculté "réalisante», les images tendant, d'elles-mêmes à s'imposer à s'imposer à nous et à se donner pour réelles. Cette théorie issue du cartésianisme, se retrouve dans les philosophies pourtant opposées, de Taine à Bergson» (Alquié, 1955, 170).

Ferdinand Alquié est une voix esseulée en ce qui concerne la mise en évidence des paradoxes du mouvement. ${ }^{15} \mathrm{Il}$ a proposé de même une explication de ce que l'œuvre de Breton est, en fait, une formule de binôme :

13 Plus concrètement: On opère le transfert de la nominalisation qui passe de la chose nommée par le vocable qui nomme au système d'une langue concrète : le contenu que présuppose la réalité extralinguistique est désormais à la portée des interlocuteurs de la même communauté linguistique.

14 Ferdinand Alquié considérait le surréalisme comme une sorte de philosophie.

15 Il faudrait mentionner aussi Maurice Nadeau qui évoque le caractère rationnel du mouvement en définissant l'époque entre 1925 et 1930 dans le sens de » la période raisonnante du surréalisme «. Cf : Nadeau, Maurice : «La Période raisonnante du surréalisme ». In : L'Histoire du surréalisme. Paris : Seuil, 1964, 95. 
«Breton, au contraire, ne cède jamais tout à fait à l'irrationalité des images. Le contrôle de la lucidité, les normes morales de la signification des œuvres lui importent toujours. D'aucune production automatique in ne renonce à découvrir le sens ; aux richesses de l'inconscient, il veut joindre les lumières de la conscience» (Alquié 1955, 177).

L'image doit être reliée à l'esprit : elle en est le produit précieux. Pierre Reverdy met le lien entre l'image et l'esprit en tête de son texte sur l'image poétique : "L'image est une création pure de l'esprit» (Reverdy 1918, 3). Académie française définit l'esprit comme "Principe de pensée»" ${ }^{16}$ et elle rajoute : "Faculté de comprendre, intelligence, intellect» ${ }^{17}$ et : "Forme particulière de l'intelligence» ${ }^{18}$. Comme une des cooccurrences sur la liste des emplois possibles le dictionnaire propose la phrase : "Les Français ont la réputation d'avoir l'esprit cartésien»" ${ }^{19}$. L'image surréaliste émane donc de la même faculté que le raisonnement puisque l'esprit et l'intelligence ou la raison sont synonymes. C'est ce qui est sous-entendu dans l'extrait suivant :

»Si les profondeurs de notre esprit recèlent d'étranges forces capables d'augmenter celles de la surface, ou de lutter victorieusement contre elles, il y a tout intérêt à les capter, à les capter d'abord, pour les soumettre ensuite, s'il y a lieu, au contrôle de notre raison. Les analystes eux-mêmes n'ont qu'à y gagner. Mais il importe d'observer qu'aucun moyen n'est désigné a priori pour la conduite de cette entreprise, que jusqu'à nouvel ordre elle peut passer pour être aussi bien du ressort des poètes que des savants et que son succès ne dépend pas des voies plus ou moins capricieuses qui seront suivies «(Breton 1924, 5).

Le jugement d'André Breton se trouve corroboré par l'analyse à laquelle passe Marcel Raymond dans son livre présentant la figure de référence sur le sujet :

«Notons que ces images, plus ou moins absurdes logiquement, plus ou moins déconcertantes pour les sens, en raison de leur étrangeté, engendrent cependant de représentations sensibles ; des feux-follets bondissent de-ci, de-là, des irradiations, des ondes hertziennes se propagent dans la nuit. Lesprit, quelque précaution qu'il prenne pour s'isoler, ne peut faire qu'il ne soit nourri d'éléments venus du monde extérieur ; il ne peut raconter son histoire, si intérieure soit-elle, qu'en empruntant des formes qui ont des corps et qui portent des noms» (Raymond 1940, 287).

Les images surréalistes quoi que veuillent leurs auteurs se ploient à la logique qui règle les actes de parole ou les assertions que l'on se propose afin de former

16 Cf: https://www.dictionnaire-academie.fr/article/A9E2642, consulté le 27 juillet 2019.

17 Ibidem.

18 Ibidem.

19 Ibidem. 
un message. Afin de transmettre les «représentations sensibles» ${ }^{20}$ les surréalistes recourent à l'arsenal qui fait parti du canon de la poésie en tant que telle : "Image, image poétique ou métaphore sont synonymes aux yeux des surréalistes qui d'ailleurs ne retiennent pas la distinction scolaire entre comparaison et métaphore» (José 1973, 90). Le surréalisme ne recule ni devant l'analogie qui est par son origine un outil philosophique puisqu'elle concerne la répartition de l'être. ${ }^{21} \mathrm{Et}$ même lorsque les surréalistes se proposent d'interpréter les entités opaques de leur imaginaire, ils retombent dans l'aporie, car ils sont obligés de se servir de la logique formelle à laquelle ils ont contesté la suprématie pour ne pas dire la valeur. Ils ne peuvent sortir de l'impasse sans avoir au préalable rétabli l'objet dans sa forme initiale :
«À la faveur de ces identités élémentaires, dit Eluard, de nouvelles images, plus arbitraires parce que formelles, se composent : le bocal de ta voix, les tiroirs du vallon ${ }^{22}$. Plus précisément, il faudrait dire que l'imagination opère ici en pas- sant d'un objet à un autre objet voisin dans l'espace (poisson $\rightarrow$ bocal, langue $\rightarrow$ voix, argenterie $\rightarrow$ tiroirs, ruisseau $\rightarrow$ vallon) : procès métonymique; puis la syntaxe associe - de manière «formelle» dit Eluard - bocal et vallon, comme s'associaient par analogie sémantique partielle, poisson et langue, argenterie et ruisseau» (Abastado 1971, 86).

L'anagogie des images surréalistes n'est possible qu'à l'intérieur d'un système des signes qui est celui dont l'exégèse littéraire et philosophique se sert lors de l'analyse de tout texte. Le surréalisme - malgré le charme discret de son apriorisme - n'est pas une exception qui confirmerait la règle. Il confirme la règle, sans exception.

L'imaginaire surréaliste se laisse expliquer par les termes de la syntaxe et logique quotidienne. Prenant deux exemples de l'art du mouvement, Le Grand Masturbateur ${ }^{23}$ et L'Âge d'or. ${ }^{24}$ On donne volontiers la trame du récit que fournissent les données encyclopédiques à propos de L'Âge d'or. Le contenu du film abonde de réseaux des significations prélevées sur la vie du quotidien. A-t-on vu quelque chose de plus dépourvu d'originalité que l'histoire d'un couple dont le bonheur (c'est-à-dire, la liberté sexuelle) est menacé par l'entourage familial ? Pour faire l'écho les auteurs durent recourir au scandale, aux messageries roses d'un érotomane : le marquis de Sade faisait l'objet d'une lecture illégale à l'époque : on

20 Cité supra.

21 Cf: Przywara, Erich. Analogia entis. Einsideln : Johannes-Verlag, 1962.

22 L'auteur analyse le poème «Ta langue, le poisson rouge dans le bocal de ta voix » in : Apollinaire, Guillaume. Euvres poétiques. Paris : Gallimard, 1965, p. 56 et «Les Reposoirs de la procession ». In : https:// fr.wikisource.org/wiki/Les_Reposoirs_de_la_procession_(1893)/Tome_I, consulté le 28 juillet 2019.

23 Cf : https://fr.wikipedia.org/wiki/Le_Grand_Masturbateur, consulté le 30 juillet 2019.

24 Cf: https://fr.wikipedia.org/wiki/L\%27\%C3\%82ge_d\%27or_(film,_1930), consulté le 29 juillet 2019. 
n'avait d'accès à ses textes que sur autorisation, ce qui n'est plus le cas. La mise en œuvre des "pionniers» cinématographiques de la provenance surréaliste pourrait aujourd'hui paraître sans intérêt, la clientèle «assagie» qu'un tel film enthousiasmait à l'époque a disparu : celle d'actualité préférerait jeter son dévolu sur des sujets plus scabreux. L'Áge d'or laisse de plus en plus l'auditoire sur sa faim :

«Histoire de la communion totale mais éphémère de deux amants que séparent les conventions familiales et sociales et les interdits sexuels et religieux, le film est une succession d`épisodes allégoriques teintés d`humour noir, commençant par un documentaire sur les scorpions et srachevant sur une transposition

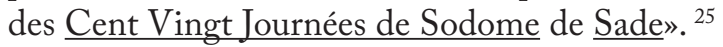

En ce qui concerne Le Grand Masturbateur, la toile est une allégorie de l'auteur lui-même. Son exégèse serait moins difficile que celle d'un $E_{s p o l i o}{ }^{26}$ qui offre une gamme d'interprétations, la majorité d'elle, depuis la découverte de Maurice Barrès (Barrès 1994, 511), allant dans les sens différents et contradictoires.

Il ne peut pas en être autrement, puisque l'unique «surréalité» dont dispose le genre homo sapiens sapiens sont la logique, l'intelligence et la raison. Tout le reste n'est que du bluff.

\section{BIBLIOGRAPHIE}

Abastado, Claude. Introduction au Surréalisme. Paris : Bordas, 1971. Alquié, Ferdinand. Philosophie du Surréalisme. Paris : Flammarion, 1955.

Apollinaire, Guillaume. Euvres poétiques. Paris : Gallimard, 1965. Aragon, Louis. Le Paysan de Paris. Paris : Gallimard, 1928.

Barrès, Maurice. «El Greco et le Secret de Tolède». In : Maurice Barrès, romans et voyages. Paris : Robert Laffont, 1994.

Benveniste, Emile. Problèmes de linguistique générale. Paris : Gallimard, 1966.

Breton, André. Le Manifeste du Surréalisme. Paris : Sagittaire, 1924.

Cassin, Barbara. "Quand lire, c'est faire ». In : Parménide : Sur la nature ou sur l'étant. Paris : Seuil, 1998.

Descartes, René. Discours de la Méthode. Paris : Flammarion, 1966.

Descartes, René. Méditations métaphysiques. Paris : Flammarion, 2011.

25 Ibidem

26 Cf:https://salvagefragments.wordpress.com/2016/06/30/earle-birneys-el-greco-espolio/, consulté le 31 juillet 2019 . 
Heidegger,Martin. Introduction à la métaphysique (trad. G. Kahn). Paris : Gallimard, 1967.

José, Pierre. Le Surréalisme. Paris : Hazan, 1973.

Légoutière, Edmond. Le Surréalisme. Paris : Masson et Cie, 1972.

Masson, André. Le Rebelle du surréalisme. Paris : Hermann, 1976.

Nadeau, Maurice. «La Période raisonnante du surréalisme ». In : L'Histoire du surréalisme. Paris : Seuil, 1964.

Naville, Pierre. La Révolution et les Intellectuels. Paris : Gallimard, 1975.

Przywara, Erich. Analogia entis. Einsiedeln : Johannes-Verlag, 1962.

Raymond, Marcel. De Baudelaire au Surréalisme. Paris : José Corti, 1940.

Reverdy, Pierre. «L'image est une création pure de l'esprit». In : Nord-Sud. Paris : Reverdy (éditeur), 1918.

Saussure, Ferdinand de. Cours de Linguistique générale. Paris : Payot, 1922.

\section{SOURCES INTERNET}

https://www.dictionnaire-academie.fr/article/A9I0182, consulté le 27 juillet, 2019.

https://fr.wikisource.org/wiki/Les_Reposoirs_de_la_procession_(1893)/Tome_I, consulté le 28 juillet 2019 .

https://fr.wikipedia.org/wiki/L\%27\%C3\%82ge_d\%27or_(film,_1930), consulté le 29 juillet 2019.

https://fr.wikipedia.org/wiki/Le_Grand_Masturbateur, consulté le 30 juillet 2019.

https://salvagefragments.wordpress.com/2016/06/30/earle-birneys-el-greco-espolio/, consulté le 31 juillet 2019.

Boštjan Marko Turk

Université de Ljubljana

Bostjan-marko.turk@guest.arnes.si

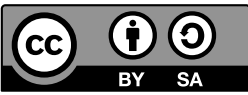

\section{Protislovja nadrealizma}

Ta študija se ukvarja $z$ vprašanjem aporij, ki zadevajo nadrealistično pojmovanje jezika in njegovih pesniških orodij. Nadrealisti so močno nasprotovali pojmovanju, da je to, kar obstaja, razumu dostopno in zato za razumljivo. Trdili so, da obstaja sfera onkraj realnosti, $\mathrm{v}$ katero razum ni sposoben prodreti. A vsa orodja in imaginarij njihove poetične govorice 
so bila zasnovana na elementih razuma, četudi si tega niso hoteli priznati in so razumu odrekali sleherno legitimnost. Študija prikazuje, kako so bila njihova teoretična utemeljevanja lastne poetike $\mathrm{v}$ esencialnem smislu protislovna.

Ključne besede: nadrealizem, razum, misel, duh, Descartes, Voltaire, Breton, jezik, jezikoslovje, znak, Benveniste, Saussure, protislovje.

\section{Abstract}

The present study addresses the problem of the aporias which concern the surrealist conception of language and its poetic tools. The surrealists opposed to the mindset which supposes that what exists has its raison dêtre and can - therefore - be considered intelligible. They postulated that there is a sphere beyond reality in which reason is not able to penetrate. But all the tools that the surrealists proposed in order to penetrate to a higher sphere of reality were in fact designed out of the modalities of reasoning.

Key words: surrealism, reason, thought, spirit, Descartes, Voltaire, Breton, language, linguistics, sign, Benveniste, Saussure, antinomy. 